\title{
Susceptibility of transient chimera states
}

\author{
Thomas Lilienkamp ${ }^{1,2, *}$ and Ulrich Parlitz ${ }^{1,2,3}$ \\ ${ }^{1}$ Max Planck Institute for Dynamics and Self-Organization, Am Fassberg 17, 37077 Göttingen, Germany \\ ${ }^{2}$ German Center for Cardiovascular Research (DZHK), Partner Site Göttingen, Robert-Koch-Straße 42a, 37075 Göttingen, Germany \\ ${ }^{3}$ Institut für Dynamik komplexer Systeme, Georg-August-Universität Göttingen, Friedrich-Hund-Platz 1, 37077 Göttingen, Germany
}

(Received 11 December 2019; accepted 26 August 2020; published 21 September 2020)

\begin{abstract}
Chaotic dynamics of a dynamical system is not necessarily persistent. If there is (without any active intervention from outside) a transition towards a (possibly nonchaotic) attractor, this phenomenon is called transient chaos, which can be observed in a variety of systems, e.g., in chemical reactions, population dynamics, neuronal activity, or cardiac dynamics. Also, chimera states, which show coherent and incoherent dynamics in spatially distinct regions of the system, are often chaotic transients. In many practical cases, the control of the chaotic dynamics (either the termination or the preservation of the chaotic dynamics) is desired. Although the self-termination typically occurs quite abruptly and can so far in general not be properly predicted, previous studies showed that in many systems a "terminal transient phase" (TTP) prior to the self-termination existed, where the system was less susceptible against small but finite perturbations in different directions in state space. In this study, we show that, in the specific case of chimera states, these susceptible directions can be related to the structure of the chimera, which we divide into the coherent part, the incoherent part and the boundary in between. That means, in practice, if self-termination is close we can identify the direction of perturbation which is likely to maintain the chaotic dynamics (the chimera state). This finding improves the general understanding of the state space structure during the TTP, and could contribute also to practical applications like future control strategies of epileptic seizures which have been recently related to the collapse of chimera states.
\end{abstract}

DOI: 10.1103/PhysRevE.102.032219

\section{INTRODUCTION}

In systems which show chaotic transients an apparently chaotic dynamics can be observed for a certain amount of time [characterized by positive (finite time) Lyapunov exponents and the sensitive dependence of initial conditions], until a transition towards a possibly nonchaotic attractor occurs. In contrast to persistent chaos whose state space structure is determined by a chaotic attractor, chaotic saddles are often the underlying structure of transient chaos [1,2]. Chaotic transients appear in diverse fields, e.g., in population dynamics [3], ecology [4,5] (underlying chaotic saddle discussed in [6]), coupled FitzHugh-Nagumo oscillators [7], nuclear magnetic resonance (NMR) lasers [8], turbulence [9], neural networks [10-12], cardiac dynamics [13], or plankton blooms [14]. In this study we discuss the dynamics of chimera states, defined by the coexistence of spatial regions with coherent and incoherent dynamics. They can be found in many reallife systems, like in superconducting quantum interference devices (SQUIDS) $[15,16]$, the cognitive organization of the

\footnotetext{
*thomas.lilienkamp@ds.mpg.de
}

Published by the American Physical Society under the terms of the Creative Commons Attribution 4.0 International license. Further distribution of this work must maintain attribution to the author(s) and the published article's title, journal citation, and DOI. Open access publication funded by the Max Planck Society. brain $[17,18]$, in biological systems like self-propelled particles [19], or neural networks [20,21]. In addition, chimera states were found in quantum mechanics [22] or mechanical oscillators [23]. In 2011, Wolfrum and Omel'chenko showed [24] that their results indicated that chimera states may also be a transient phenomenon.

In many systems with transient chaos, the transition between the chaotic dynamics and the final attractor is of high interest for many reasons, in particular regarding the potential control of the dynamics (either the termination or the preservation of the chaotic dynamics). Often, the upcoming self-termination of the chaotic dynamics (without any external interaction) is not visible in conventional observables a reasonable amount of time before the transition, and therefore it is currently not clear whether a prediction of the end of a chaotic episode is, in principle, possible. However, in $[25,26]$ it was shown in spatially extended systems and low-dimensional maps that the transition from the chaotic regime towards the (nonchaotic) attractor of the system is a process with a finite length, called the "terminal transient phase" (TTP), which manifests in a change of the state space structure. During this final phase, the system is susceptible against small but finite perturbations only in specific directions.

This means in the case of spatially extended systems that before self-termination the system can only be perturbed significantly (such that self-termination is prevented) in specific regions of the spatial domain. While in $[25,26]$ these regions could only be empirically identified, we show in this study that 


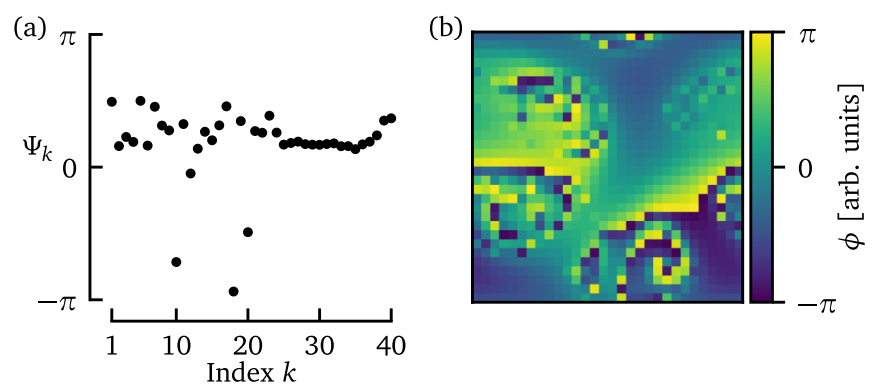

FIG. 1. Exemplary snapshots of chimera states (a) in a onedimensional ring of oscillators [Eq. (1)] with $N=40$, and the (b) two-dimensional system [Eq. (3)] for system size $A=3 \times 3=$ 9 arb. units (discretized on a square grid of $30 \times 30$ oscillators). In both cases spatially separated regions of coherent and incoherent dynamics of the phase oscillators exist simultaneously.

for the case of transient chimera states [one-dimensional (1D) and two-dimensional (2D)] these regions have a direct relation to the actual state of the system (they relate to the distinction between coherent and incoherent parts of the chimera state and the boundary region between those). In [27] Yao et al. investigated in general how $1 \mathrm{D}$ chimera states react to local perturbations of finite amplitude. However, with the relation mentioned above we suggest in this study regions of the chimera state where small but finite perturbations will most likely cause a prevention of the upcoming self-termination, if it is close to the collapse. The localized (in space and time) application of a finite perturbation is therefore in contrast to former control strategies of chimera states, which mostly rely on feedback control or other (global) approaches [28-31].

\section{MODELS}

In this study we investigate two systems of coupled phase oscillators on a 1D and 2D extended domain, respectively, using periodic boundary conditions in both cases.

In the $1 \mathrm{D}$ ring of oscillators, the temporal evolution of the phase $\Psi_{k}$ of oscillator $k$ is determined by [24]

$$
\frac{\partial \Psi_{k}(t)}{\partial t}=\omega-\frac{1}{2 R} \sum_{j=k-R}^{k+R} \sin \left[\Psi_{k}(t)-\Psi_{j}(t)+\alpha_{1 \mathrm{D}}\right]
$$

where we chose $R=0.35 N$ (with $N$ the number of phase oscillators, and indices $j$ are considered modulo $N$ ), the phase lag parameter [32] $\alpha_{1 \mathrm{D}}=1.46$, and a vanishing natural frequency $\omega=0$. Equation (1) was solved using a fourth order Runge-Kutta method with $d t=0.1$. The order parameter $Z_{1 \mathrm{D}}(t)$ reads as follows:

$$
Z_{1 \mathrm{D}}(t)=\left|\frac{1}{N} \sum_{k=1}^{N} e^{i \Psi_{k}(t)}\right|
$$

An exemplary chimera state of the system is shown in Fig. 1(a) with $N=40$ oscillators [1]. See Supplemental Material at [33] for exemplary transient episodes of a 1D and $2 \mathrm{D}$ chimera. The episodes shown here are also discussed in Figs. 3 and 4, respectively.
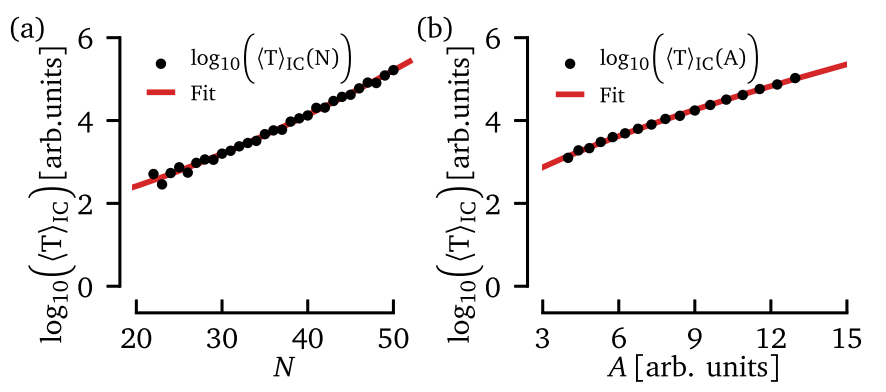

FIG. 2. The logarithms of the average transient lifetime $\langle T\rangle_{\text {IC }}$ for varying system sizes in (a) the one-dimensional system and (b) the two-dimensional system, respectively. In both cases, the average lifetimes for single domain sizes were determined via a nonlinear least-squared fit, shown in Fig. 6. The resulting average lifetimes are shown here, where the error bars are too small to be visible. They are fitted regarding Eq. (6) (with a nonlinear least-squared regression). The fit parameters are given in Table I.

In the 2D case, we use the model discussed in [34]

$$
\begin{aligned}
\frac{\partial \phi(\mathbf{x}, t)}{\partial t}= & -\int_{\mathbb{T}^{2}} G\left(\left|\mathbf{x}-\mathbf{x}^{\prime}\right|\right) \\
& \times \sin \left[\phi(\mathbf{x}, t)-\phi\left(\mathbf{x}^{\prime}, t\right)+\alpha_{2 \mathrm{D}}\right] d \mathbf{x}^{\prime},
\end{aligned}
$$

with the phase lag parameter $\alpha_{2 \mathrm{D}}=0.38 \pi$. The integration is performed over a flat torus equivalent to the square $A=$ $L \times L$ with periodic boundary conditions. To achieve transient (rather than persistent) chimera dynamics we modified the coupling of the phase oscillators by introducing a coupling range $\sigma=0.3$ :

$$
G\left(\left|\mathbf{x}-\mathbf{x}^{\prime}\right|\right)=\frac{1}{\pi} \exp \left(-\frac{\left|\mathbf{x}-\mathbf{x}^{\prime}\right|^{2}}{\sigma^{2}}\right)
$$

We solved Eq. (3) on a 2D quadratic domain (with size $A=L \times L$ ) using a fourth order Runge-Kutta method with temporal step size $d t=0.5$ and a grid spacing of $h=0.1$.

The order parameter $Z_{2 \mathrm{D}}(t)$ can be defined via a local order parameter $Z_{2 \mathrm{D}}(t)=\frac{1}{A} \int Z_{2 \mathrm{D}}^{\mathrm{loc}}(\mathbf{x}, t) d \mathbf{x}$ which is given by

$$
Z_{2 \mathrm{D}}^{\mathrm{loc}}(\mathbf{x}, t)=\left|\frac{1}{\mathcal{N}} \int_{\mathbb{T}^{2}} G\left(\left|\mathbf{x}-\mathbf{x}^{\prime}\right|\right) e^{i \phi\left(\mathbf{x}^{\prime}, t\right)} d \mathbf{x}^{\prime}\right|
$$

where $\mathcal{N}$ is a normalization constant [ $\operatorname{such}$ that $Z_{2 \mathrm{D}}^{\text {loc }}(\mathbf{x}, t)=1$ for a uniform $\phi\left(\mathbf{x}^{\prime}, t\right)$ ]. Due to periodic boundary conditions the integration domain is a $2 \mathrm{D}$ torus $\mathbb{T}^{2}$ which is discretized to enable numerical integration. An exemplary chimera state of the system is shown in Fig. 1(b) for $L=3$ (30 oscillators in each direction).

The condition for the end of the chaotic episode is chosen to be $Z_{1 \mathrm{D}}(t)>0.95$ for the $1 \mathrm{D}$ case (average value during chaotic episodes was $\left.\left\langle Z_{1 \mathrm{D}}\right\rangle \approx 0.76 \pm 0.05\right)$.

In the $2 \mathrm{D}$ system, the dynamics can either self-terminate to the globally coherent state, or to plane waves. To cover both cases, we defined self-termination if the standard deviation of $Z_{2 \mathrm{D}}(t)$ during a time interval of $\tau=5 \mathrm{arb}$. units is $<0.0001$. 

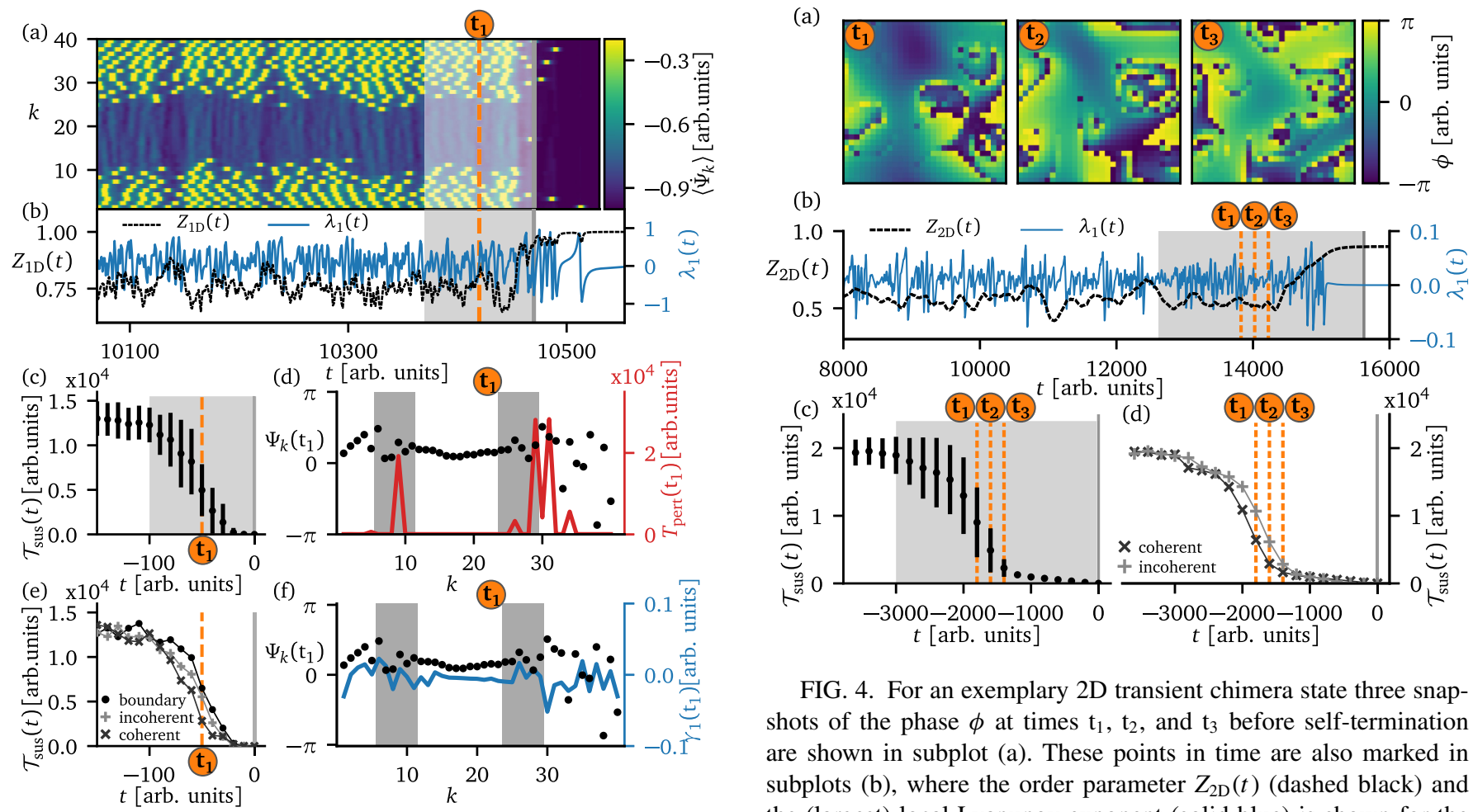

FIG. 3. The averaged phase velocity during the final episode of an exemplary chimera state (1D) is shown in subplot (a) [selftermination occurs at $t=10470$ arb.units, vertical (gray) line], with the associated order parameter $Z_{1 \mathrm{D}}(t)$ (dashed black) and the local Lyapunov exponent $\lambda_{1}(t)$ (solid blue) in subplot (b). The length of the TTP is in (a) and (b) depicted as the (gray) shaded region. In (c) and (e) $\mathcal{T}_{\text {sus }}(t)$ is plotted, where the average was taken over all perturbations (c) and over perturbations associated to coherent $(\times)$, incoherent $(+)$ and boundary regions $(\bullet)$, in (e), respectively. The error bars in (c) are standard deviations due to averaging over different initial conditions [Eq. (7), for the sake of clarity not shown in (e)]. Subplots (d) and (f) both depict the state of the chimera (black dots) at time $\mathrm{t}_{1}$ during the TTP (also marked in the other subplots), together with the distribution of $T_{\text {pert }}\left(\mathrm{t}_{1}\right)$ [solid red, subplot (d)] and the Lyapunov vector $\gamma_{1}$ which corresponds to the largest Lyapunov exponent. The boundary regions of the chimera state in (d) and (f) are marked as the (gray) shaded areas. Note, that while subplots (a), (b), (d), and (f) show results regarding a single exemplary trajectory, subplots (c) and (e) show quantities which are averaged over 50 initial conditions.

\section{RESULTS}

\section{A. Determining average lifetime of transients}

In both systems, the appearance of chimera states was transient, thus after some time the dynamics collapsed to the globally coherent state or traveling waves (in the 2D case). Whereas this transient behavior was known and investigated for systems of 1D coupled oscillators [24,35,36], with the proper setting of $\alpha_{2 \mathrm{D}}$ and the adaption of the coupling $\sigma$ we could observe transient behavior also on two dimensional spatial domains [33]. In 2D the dynamics is characterized by spatial like waves rotating around meandering incoherent regions. See Supplemental Material at [33] for exemplary transient episodes of a 1D and 2D chimera. The episodes

FIG. 4. For an exemplary 2D transient chimera state three snapshots of the phase $\phi$ at times $t_{1}, t_{2}$, and $t_{3}$ before self-termination are shown in subplot (a). These points in time are also marked in subplots (b), where the order parameter $Z_{2 \mathrm{D}}(t)$ (dashed black) and the (largest) local Lyapunov exponent (solid blue) is shown for the same initial condition shown in (a). The TTP is depicted in (b) as the gray shaded area. In (c) and (d) $\mathcal{T}_{\text {sus }}$ is plotted, where the average was taken over all perturbations (c) and over perturbations associated to coherent $(\times)$, and incoherent $(+)$ regions, in (d), respectively. The error bars in (c) are standard deviations due to averaging over different initial conditions [Eq. (7), for the sake of clarity not shown in (d)]. This distinction between coherent and incoherent parts of the chimera state is discussed in Fig. 5. Note that, while subplots (a) and (b) show results regarding a single exemplary trajectory, subplots (c) and (d) show quantities which are averaged over 20 initial conditions.

shown here are also discussed in Figs. 3 and 4, respectively. Spiral wave chimeras were also observed in an experimental setup of coupled chemical oscillators [37].

We measured for the 1D and 2D systems the average transient lifetime $\langle T\rangle_{\text {IC }}$ for varying domain sizes (the subscript IC indicates the average over many initial conditions). For this purpose, the number of oscillators was varied (technical details are given in the Appendix). The exponential scaling of $\langle T\rangle_{\text {IC }}$ with the system size shown for both cases in Fig. 2 indicates that the dynamics belongs to the group of type-II supertransients (according to [38]). The transient lifetime in these systems typically grows with:

$$
\log _{10}\left(\langle T\rangle_{\mathrm{IC}}\right) \sim(\tilde{a} X)^{\gamma}
$$

where $X=N$ for the 1D ring of oscillators Eq. (1), and $X=A$ for the 2D model Eq. (3). The fit parameters for both models are given in Table I.

In $[1,38,39]$, type-II supertransients are described with an "abrupt" transition to the (non)chaotic attractor, quantified, for example, by Lyapunov exponents or other observables. In systems with type-I supertransients, these quantities decrease rather gradually over time. In addition to the exponential 
TABLE I. Fit parameters regarding Eq. (6).

\begin{tabular}{lcc}
\hline \hline & $\tilde{a}$ [arb. units] & $\gamma$ [arb. units] \\
\hline 1D & $0.046 \pm 2.6 \times 10^{-5}$ & $1.55 \pm 0.02$ \\
$2 \mathrm{D}$ & $0.46 \pm 0.013$ & $0.68 \pm 0.004$ \\
\hline \hline
\end{tabular}

dependence of $\langle T\rangle_{\mathrm{IC}}$ on the system size, in the investigated systems "abrupt" transitions from the chaotic regime to the (possibly) nonchaotic attractor can be observed via the temporal evolution of Lyapunov exponents or the order parameter $Z_{1 \mathrm{D} / 2 \mathrm{D}}(t)$. In particular, the timescale of change of the (local) Lyapunov exponent $\tau_{\text {change }}$ (quantified by the temporal period associated with the maximum of a Fourier frequency spectrum), is much smaller than the average transient lifetime $\langle T\rangle_{\text {IC }}$. For example, $\tau_{\text {change }}^{1 \mathrm{D}} \approx 4.8 \mathrm{arb}$. units $\ll 1.33 \times$ $10^{4}$ arb. units $\approx\langle\mathrm{T}\rangle_{\mathrm{IC}}$ (for a domain size of $N=40$ ) and $\tau_{\text {change }}^{2 \mathrm{D}} \approx 150.9$ arb. units $\ll 1.75 \times 10^{4} \approx\langle\mathrm{T}\rangle_{\text {IC }}$ (for a domain size of $A=30 \times 30$ ).

\section{B. Identification of a TTP}

In the following we consider a fixed domain size $[N=$ 40 (1D) and $A=3 \times 3$ hence $30 \times 30$ oscillators (2D)]. As a next step we randomly chose several initial conditions and applied small but finite localized perturbations (amplitude $\Delta=0.1$ for both systems) during the phase just before self-termination. Note that the perturbations were applied to trajectories just before self-termination. With this approach, we wanted to identify those perturbations which can prevent the upcoming self-termination. In detail, perturbations were applied at single oscillators independently and the resulting average lifetime of the perturbed trajectory was measured beginning with the point in time when the perturbation was applied (only one perturbation at a time was performed). That means, in the one-dimensional case we independently applied 40 perturbations, leading to 40 perturbed trajectories with new lifetimes $T_{k}$ where $k=\{1, \ldots, 40\}$. Hence, $30 \times 30=900$ perturbations were applied in the $2 \mathrm{D}$ model. The average taken over all perturbed trajectories $\langle T\rangle_{\text {pert }}=$ $\frac{1}{N} \sum_{k} T_{\text {pert }}^{k}$ describes then how susceptible the state is against finite perturbations (at the given point in time). Since $\langle T\rangle_{\text {pert }}$ so far describes only the state space structure regarding a single trajectory, the procedure was performed for many initial conditions, and $\langle T\rangle_{\text {pert }}$ was additionally averaged over the investigated initial conditions (time was normalized such that the temporal points of termination of all trajectories coincide):

$$
\mathcal{T}_{\text {sus }}(t)=\left\langle\langle T(t)\rangle_{\text {pert }}\right\rangle_{\mathrm{IC}} .
$$

In the following section, it will be demonstrated that the derived quantity $\mathcal{T}_{\text {sus }}(t)$ describes how susceptible the system is against finite perturbations. With the algorithm described above, the existence of a TTP (finite amount of time before self-termination where the system reacts significantly different against finite perturbations than during earlier phases) could be verified in many systems (cardiac tissue models, neuronal models, low-dimensional maps) [25,26].

We could determine a TTP also in the investigated systems of coupled phase oscillators. In Fig. 3(a) a typical trajectory of the 1D system is shown (the averaged phase velocity $\left\langle\dot{\Psi}_{k}\right\rangle$, details can be found in the Appendix) during the final phase before self-termination ( $t_{\text {term }} \approx 10470$ arb. units). Similarly, Fig. 4(a) shows three snapshots $\left(t_{1}, t_{2}\right.$, and $\left.t_{3}\right)$ of $\phi$ during the final phase of a typical trajectory in the 2D system. In both cases the upcoming self-termination is not obviously visible in quantities like the order parameter $Z_{1 \mathrm{D} / 2 \mathrm{D}}(t)$ or the largest local Lyapunov exponent $\lambda_{1}(t)$ [dashed black and solid blue in Figs. 3(b) and 4(b), respectively] a significant amount of time beforehand. The collapse seems to be rather abrupt.

However, by computing $\mathcal{T}_{\text {sus }}$ during the phase before selftermination we can show that the TTP is also present in both systems of oscillators. In Figs. 3(c) and 4(c) $\mathcal{T}_{\text {sus }}$ is shown (averaged over 50 and 20 initial conditions for 1D and $2 \mathrm{D}$, respectively). In both cases, $\mathcal{T}_{\text {sus }}$ decreases a significant amount of time before the self-termination, meaning that when time approaches self-termination $(t=0)$, fewer and fewer perturbations can significantly change the reference trajectory anymore. The approximate temporal length of the TTP (which depends also on the perturbation amplitude $\Delta$ $[25,26])$ is then defined by the interval from the beginning of the decrease of $\mathcal{T}_{\text {sus }}$ until the point of self-termination. We could therefore identify the length of the TTP in both cases $\mathrm{TTP}_{1 \mathrm{D}}=100$ arb. units and $\mathrm{TTP}_{2 \mathrm{D}}=3000$ arb. units [additionally marked as a (gray) shaded region in Figs. 3(a) and 3(b) (1D), and Fig. 4(b) (2D)]. Thereby the temporal length is significantly longer than the Lyapunov time $\tau_{1 \mathrm{D}}^{L} \approx$ 12.46 arb. units and $\tau_{2 \mathrm{D}}^{L} \approx 116.2$ arb. units (where $\tau^{L}=\frac{1}{\lambda_{1}}$ ).

This means that the state space structure in the vicinity of the reference trajectory is different in regions close to exits from the chaotic regime. The underlying mechanism for the decrease of $\mathcal{T}_{\text {sus }}$ is that perturbations in specific directions (applied to specific oscillators) have basically no relevant effect concerning the upcoming self-termination. That means parts of the system are nonsusceptible for finite perturbations. Examples for both systems are shown in Figs. 3(d) and 5(b) where $\mathcal{T}_{\text {sus }}$ is shown for specific points in time before selftermination ( $t_{1}$ in the $1 \mathrm{D}$ case, $t_{1}, t_{2}$, and $t_{3}$ in the $2 \mathrm{D}$ case) of an exemplary trajectory.

\section{Identification of (non)susceptible regions}

The existence of a TTP and related spatially connected regions of (non)susceptibility have been shown for other spatially extended systems previously $[25,26]$, but the emerging patterns of these regions could not be related to the actual state of the system. However, in the particular case of transient chimera states, we find a relation between the state of the chimera and its "vulnerability" against small perturbations (when the system is close to self-termination). Specifically, we categorize the 1D chimeras into a coherent part, an incoherent part, and the boundary between those regions (in 2D we distinguish only between a coherent and an incoherent regime). We determined these regions by using the averaged phase velocity $\left\langle\dot{\Psi}_{k}\right\rangle$ (1D) and in 2D the local order parameter defined in Eq. (5) [shown in the 2D case in Fig. 5(a) for three points in time]. More details about the categorization of different parts are given in Appendix C.

In the $1 \mathrm{D}$ system, we observe that perturbations applied at the boundary between the coherent and the incoherent 


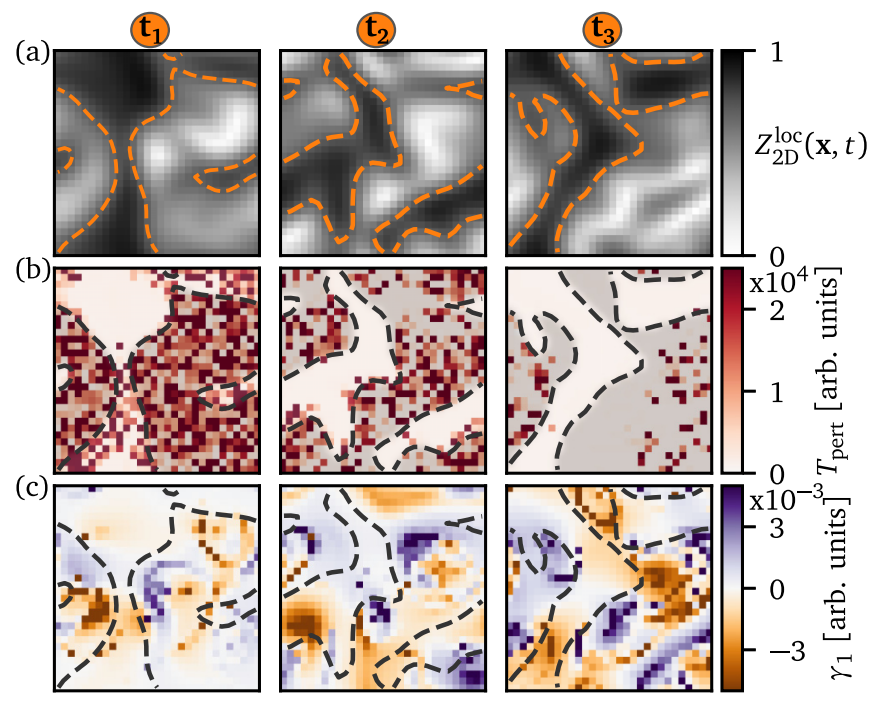

FIG. 5. The first row shows the local order parameter $Z_{2 \mathrm{D}}^{\text {loc }}(t)$ [Eq. (5)] of the system for the exemplary trajectory discussed in Fig. 4 for three points in time $t_{1}, t_{2}$, and $t_{3}$, corresponding to the snapshots of $\phi$ shown in Fig. 4(a). The dashed orange line indicates the isolines of $Z_{2 \mathrm{D}}^{\text {loc }}(t)=0.7$ which is used to distinguish coherent $\left[Z_{2 \mathrm{D}}^{\text {loc }}(t)>0.7\right]$ from incoherent regions $\left[Z_{2 \mathrm{D}}^{\text {loc }}(t)<0.7\right]$. In the second and third rows, the patterns of $T_{\text {pert }}$ [subplot (b)] and the Lyapunov vector $\gamma_{1}$ corresponding to the largest Lyapunov exponent [subplot (c)] are depicted, respectively, for the same points in time as in (a). In (a) and (b), the dashed lines separate coherent regions and incoherent regions [additionally marked by a gray shaded overlay in (b)] obtained from the isolines in (a) to compare the patterns of $T_{\text {pert }}$ and the Lyapunov vector with the coherent or incoherent parts of the chimera state.

regimes are more likely to perturb the trajectory significantly (resulting in a large $T_{\text {pert }}$ ). This is shown in Fig. 3(d), where the state of the chimera at point $t_{1}$ [marked also in Fig. 3(a)] is plotted in black. The boundary between the coherent and incoherent regions is marked by the gray shaded region. The distribution of $T_{\text {pert }}$ in Fig. 3(d) shows that when close to the point of self-termination, perturbations applied at oscillators inside the boundary region are more likely to lead to significant transient times $T_{\text {pert }}$ (in comparison to the coherent and incoherent regimes). We demonstrated statistically [by averaging over the 50 initial conditions also used in Fig. 3(c)] that there is actually a hierarchy: perturbations applied at the boundary have the highest chance of perturbing the reference trajectory significantly, whereas when they are applied at the coherent region they have the least chance [dots (boundary), $x$ in Fig. 3(e)].

In the case of infinitesimal perturbations, the direction of perturbation which will cause the largest separation of a reference trajectory is described by (covariant) Lyapunov vectors. Comparing the distribution of "significant" perturbations $T_{\text {pert }}$ [in Fig. 3(d)] with the first Lyapunov vector $\gamma_{1}$ [solid blue in Fig. 3(f)], shows some similarities (peak in right boundary), but indicates that our analysis using finite perturbations contains additional or different information than the Lyapunov approach since the left boundary region around $k \approx 12$ is not significantly recognized as a region of high susceptibility by $\gamma_{1}$.
Also in the 2D case the distribution of $T_{\text {pert }}$ (Fig. 5 for $t_{1}$, $\left.t_{2}, t_{3}\right)$ can be related to the coherent or incoherent regions of the chimera state [due to the low resolution $(30 \times 30)$ and the specific structure of the chimera state we could not properly define a boundary region here]. Coherent and incoherent regions [separated by the dashed orange line in Fig. 5(a), the points in time $t_{1}, t_{2}, t_{3}$ relate to the three snapshots of the chimera state in Fig. 4(a)] were distinguished by the local order parameter $Z_{2 \mathrm{D}}^{\text {loc }}$. In Fig. 5(b) the distribution of nonvanishing $T_{\text {pert }}$ for the exemplary trajectory mostly coincides with the incoherent regions of the chimera state (boundaries between coherent and incoherent regions are marked by dashed lines). This is quantified by $\mathcal{T}_{\text {sus }}$ which was averaged only over perturbations in coherent regions [(x) in Fig. 4(d)] or incoherent regions [(+) in Fig. 4(d)]. Similar to the 1D results, perturbing the system in the incoherent region of the chimera state is more likely to prevent self-termination if it is close. Also, the first Lyapunov vector $\gamma_{1}$ [depicted in Fig. 5(c)] shows similarities to the distribution of $T_{\text {pert }}$ [Fig. 5(b)]. However, comparable to the $1 \mathrm{D}$ results, $\gamma_{1}$ does not cover all susceptible regions (defined by nonvanishing $T_{\text {pert }}$ ) and only partly coincides with coherent and incoherent regions (separated by a dashed line).

\section{CONCLUSION}

In this study we investigated transient chaotic chimeras. In the 2D case we adapted the system of Martens et al. [34] such that it shows (coherent) waves which rotate around meandering incoherent regions.

In the 1D ring of oscillators and the 2D system, a TTP was identified, where the system is susceptible against small but finite locally confined perturbations only in specific spatial domains. The temporal length of this transition phase is much larger than the Lyapunov time, which means that the dynamics near the "exits" of the chaotic regime of the state space is determined by a specific global structure (suggesting, for instance, high-dimensional tubes).

We could furthermore show that a relation between the actual state of the system and the patterns of susceptible regions (defined by nonvanishing $T_{\text {pert }}$ ) exists: We found that if a perturbation is applied during the TTP, it is more likely to change the trajectory significantly if it is applied in the $1 \mathrm{D}$ case at the boundary between the coherent and incoherent parts of the chimera and in the 2D case at incoherent parts of the chimera. In practice we can therefore provide a suggestion for the location of a perturbation, if the system is close to self-termination and the preservation of the chaotic phase is desired. These findings could in the future influence the developments of control strategies for epileptic seizures which have recently been related to the collapse of chimera states [40]. Furthermore, the results presented in this study improve the general understanding of the mechanism and control of the transition towards the synchronized or coherent state, which may play a role in many real-life systems $[15-17,19,20,22,23]$ where chimera states were found and controlling them could become relevant in future applications. In a broad perspective, the emergence of spontaneous synchronization plays a fundamental role, e.g., in dissimilar silicon nitride micromechanical oscillators [41], where the authors suggest a new class of 


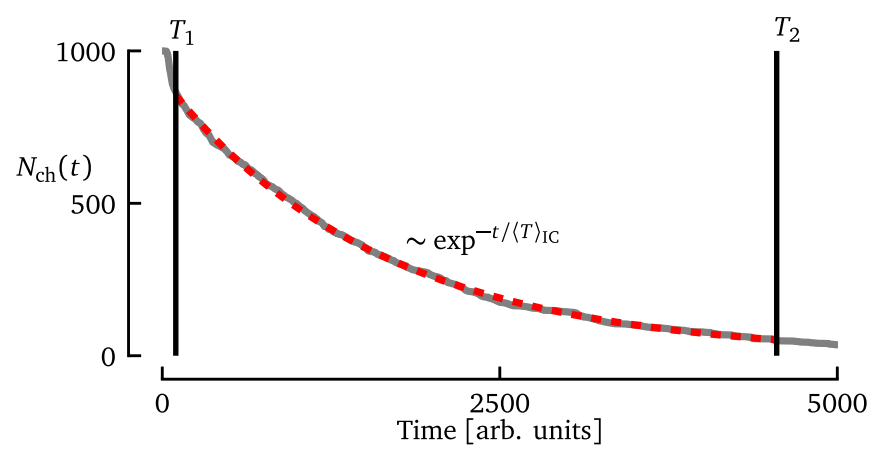

FIG. 6. In solid gray, the number of initial conditions $N_{\mathrm{ch}}(t)$, which show chaotic dynamics at time $t$ is shown, which typically decreases exponentially. The average lifetime $\langle T\rangle_{\text {IC }}$ was determined by fitting $N_{\mathrm{ch}}(t)$ (nonlinear least-squared). The fit is depicted as the dashed red line. An amount of time $T_{1}$ was discarded for the fit to separate out those initial conditions which converge to the resting state immediately [initial drop of $N_{\mathrm{ch}}(t)$ ]. Also, the fit was performed till $N_{\mathrm{ch}}(t)$ dropped below 50 initial conditions (marked by $T_{2}$ ), due to low statistics for $t>T_{2}$.

devices in sensing, signal processing, and on-chip nonlinear dynamical systems.

\section{ACKNOWLEDGMENTS}

We thank the German Centre for Cardiovascular Research (DZHK e.V.) for financial support. Furthermore, we thank Stefan Luther for fruitful discussions and continuous support.

\section{APPENDIX A: INITIAL CONDITIONS AND DETECTION OF SELF-TERMINATION}

For the analysis of how the average transient lifetime $\langle T\rangle_{\text {IC }}$ depends on the system size [results presented in Figs. 2(a) and 2(b) of the main paper] 1000 initial conditions were created for each system (1D and 2D, respectively) and for each system size $(N=[22,23, \ldots, 50]$ (1D) and $A=$ $[4.0,4.41,4.84,5.29,5.76,6.25,6.76,7.29,7.84,8.41,9.0$, $9.61,10.24,11.56,12.25,12.96]$ (2D).

In the $1 \mathrm{D}$ case, the initial phases of the oscillators were created randomly from the interval $[-\pi, \pi]$. In the $2 \mathrm{D}$ system, the quadratic domain was divided into four quarters with the initial values $[-2,-1,1,2]$. In this way, a spiral-like dynamics was initiated. For different initial conditions, the phase of each oscillators was disturbed randomly by random values from the interval $[0,1]$.

\section{APPENDIX B: DETERMINING THE AVERAGE TRANSIENT LIFETIME $\langle T\rangle_{\text {IC }}$}

The average transient lifetime $\langle T\rangle_{\text {IC }}$ for each system size was determined by creating 1000 initial conditions and then measuring the number of initial conditions $N_{\mathrm{ch}}(t)$, which show chaotic dynamics at time $t$. While $N_{\mathrm{ch}}(t)$ usually decreases exponentially in systems of type-II supertransients [1], the exponential decay rate is proportional to the average transient lifetime. Figure 6 depicts $N_{\mathrm{ch}}(t)$ for the 1D case and an exemplary system size of $N=30$.

\section{APPENDIX C: DETERMINING COHERENT, INCOHERENT, AND BOUNDARY REGIONS}

To distinguish in the 1D case between coherent parts, incoherent parts, and the boundary zone between those two regions, an averaged phase velocity was determined (time window was 50 arb. units). A value larger than -0.6 was then associated with incoherent dynamics, a lower value was associated with a coherent dynamics. In a second step, the boundary zone of a fixed number of six oscillators was defined at the transition between the coherent and incoherent parts.

\section{APPENDIX D: LYAPUNOV ANALYSIS}

The Lyapunov exponent and the Lyapunov vector corresponding to the largest Lyapunov exponent were determined by computing the evolution of small but finite perturbations (via linearized equations) along the respective trajectory. By using a QR decomposition every ten simulation steps of local Lyapunov exponents were extracted and perturbations were orthonormalized via a $\mathrm{QR}$ decomposition.

In the $2 \mathrm{D}$ case, the local order parameter $Z_{2 \mathrm{D}}^{\text {loc }}(\mathbf{x}, t)$ [Eq. (5)] was used (with the threshold $<0.7$ : incoherent, $>0.7$ : coherent).
[1] Y.-C. Lai and T. Tél, Transient Chaos: Complex Dynamics on Finite-Time Scales, Applied Mathematical Sciences No. 173 (Springer, New York, 2011).

[2] Y.-C. Lai and R. L. Winslow, Phys. Rev. Lett. 74, 5208 (1995).

[3] K. McCann and P. Yodzis, Am. Nat. 144, 873 (1994).

[4] P. Turchin and S. P. Ellner, Ecology 81, 3099 (2000).

[5] S. P. Ellner and P. Turchin, Oikos 111, 620 (2005).

[6] T. Tel, Y.-C. Lai, and M. Gruiz, Int. J. Bifurcation Chaos 18, 509 (2008).

[7] G. Ansmann, K. Lehnertz, and U. Feudel, Phys. Rev. X 6, 011030 (2016).
[8] I. M. Jánosi, L. Flepp, and T. Tél, Phys. Rev Lett. 73, 529 (1994).

[9] H. Faisst and B. Eckhardt, Phys. Rev. Lett. 91, 224502 (2003).

[10] B. Poole, S. Lahiri, M. Raghu, J. Sohl-Dickstein, and S. Ganguli, in Proceedings of the 30th International Conference on Neural Information Processing Systems (Curran Associates Inc., Red Hook, NY, USA, 2016), pp. 3368-3376.

[11] X.-S. Yang and Q. Yuan, Neurocomputing 69, 232 (2005).

[12] X. Wu and H. Liljenstrom, Network: Comput. Neural Syst. 5, 47 (1994).

[13] T. Lilienkamp, J. Christoph, and U. Parlitz, Phys. Rev. Lett. 119, 054101 (2017). 
[14] E. Hernández-García and C. López, Ecological Complexity 1, 253 (2004).

[15] N. Lazarides, G. Neofotistos, and G. P. Tsironis, Phys. Rev. B 91, 054303 (2015).

[16] J. Hizanidis, N. Lazarides, and G. P. Tsironis, Front. Appl. Math. Stat. 5, 33 (2019).

[17] K. Bansal, J. O. Garcia, S. H. Tompson, T. Verstynen, J. M. Vettel, and S. F. Muldoon, Sci. Adv. 5, eaau8535 (2019).

[18] L. Ramlow, J. Sawicki, A. Zakharova, J. Hlinka, J. C. Claussen, and E. Schöll, Europhys. Lett. 126, 50007 (2019).

[19] N. Kruk, Y. Maistrenko, and H. Koeppl, Phys. Rev. E 98, 032219 (2018).

[20] M. S. Santos, P. R. Protachevicz, K. C. Iarosz, I. L. Caldas, R. L. Viana, F. S. Borges, H.-P. Ren, J. D. Szezech, A. M. Batista, and C. Grebogi, Chaos 29, 043106 (2019).

[21] S. Majhi, B. K. Bera, D. Ghosh, and M. Perc, Phys. Life Rev. 28, 100 (2019).

[22] V. M. Bastidas, I. Omelchenko, A. Zakharova, E. Schöll, and T. Brandes, Phys. Rev. E 92, 062924 (2015).

[23] E. A. Martens, S. Thutupalli, A. Fourrière, and O. Hallatschek, Proc. Natl. Acad. Sci. 110, 10563 (2013).

[24] M. Wolfrum and O. E. Omel'chenko, Phys. Rev. E 84, 015201(R) (2011).

[25] T. Lilienkamp and U. Parlitz, Phys. Rev. Lett. 120, 094101 (2018).

[26] T. Lilienkamp and U. Parlitz, Phys. Rev. E 98, 022215 (2018).

[27] N. Yao, Z.-G. Huang, H.-P. Ren, C. Grebogi, and Y.-C. Lai, Phys. Rev. E 99, 010201(R) (2019).
[28] I. Omelchenko, T. Hülser, A. Zakharova, and E. Schöll, Front. Appl. Math. Stat. 4, 67 (2019).

[29] I. Omelchenko, O. E. Omel'chenko, A. Zakharova, M. Wolfrum, and E. Schöll, Phys. Rev. Lett. 116, 114101 (2016).

[30] J. Sieber, O. E. Omel'chenko, and M. Wolfrum, Phys. Rev. Lett. 112, 054102 (2014).

[31] G. Ruzzene, I. Omelchenko, E. Schöll, A. Zakharova, and R. G. Andrzejak, Chaos 29, 051103 (2019).

[32] H. Sakaguchi and Y. Kuramoto, Prog. Theor. Phys. 76, 576 (1986).

[33] See Supplemental Material at http://link.aps.org/supplemental/ 10.1103/PhysRevE.102.032219 for exemplary transient episodes of a 1D and 2D chimera. The episodes shown here are also discussed in Figs. 3 and 4, respectively.

[34] E. A. Martens, C. R. Laing, and S. H. Strogatz, Phys. Rev. Lett. 104, 044101 (2010).

[35] S. Rakshit, B. K. Bera, M. Perc, and D. Ghosh, Sci. Rep. 7, 1 (2017).

[36] E. Schöll, Eur. Phys. J.: Spec. Top. 225, 891 (2016).

[37] J. F. Totz, J. Rode, M. R. Tinsley, K. Showalter, and H. Engel, Nat. Phys. 14, 282 (2018).

[38] J. P. Crutchfield and K. Kaneko, Phys. Rev. Lett. 60, 2715 (1988).

[39] K. Kaneko, Phys. Lett. A 149, 105 (1990).

[40] R. G. Andrzejak, C. Rummel, F. Mormann, and K. Schindler, Sci. Rep. 6, 23000 (2016).

[41] M. Zhang, G. S. Wiederhecker, S. Manipatruni, A. Barnard, P. McEuen, and M. Lipson, Phys. Rev. Lett. 109, 233906 (2012). 